\title{
Differential diagnosis of gallbladder ascariasis debris: the added value of contrast enhanced ultrasound with high frequency transducer
}

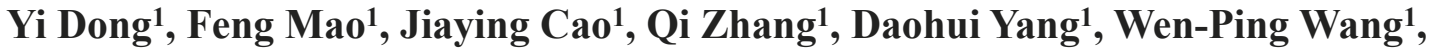 \\ Christoph F. Dietrich ${ }^{2}$
}

${ }^{1}$ Department of Ultrasound, Zhongshan Hospital, Fudan University, Shanghai, China, ${ }^{2}$ Medizinische Klinik 2, Caritas-Krankenhaus Bad Mergentheim, Bad Mergentheim

\begin{abstract}
Ascariasis debris of the gallbladder is a very rare incidental or symptomatic presentation. Ascaris debris has a pseudotumorous appearance and may be confused with neoplasia. The aim of the current retrospective study is to investigate the value of contrast enhanced ultrasound (CEUS) for the differential diagnosis of ascariasis debris and neoplasia of the gallbladder. Material and methods: Conventional B-mode ultrasound (BMUS) and CEUS were performed for solitary echo-rich gallbladder lesions. Analysis of the CEUS enhancement pattern of the lesions was conducted according to the current EFSUMB guidelines. Two radiologists assessed the CEUS enhancement patterns in consensus. The final gold standard was surgery with histological examination or imaging follow-up. Results: A total of 9 patients with final diagnoses of gallbladder ascariasis debris were included. As a control group 26 solitary hyperechoic gallbladder lesions without shadowing were included as a control group. The typical zigzag morphology with multiple echogenic parallel lines without shadow were detected inside the lumen of the gallbladder in 6 patients. After injection of $2.4 \mathrm{ml}$ ultrasound contrast agents, all hyperechoic gallbladder ascariasis debris lesions showed no enhancement. All patients in the control group with similar BMUS morphology showed contrast enhancement. Conclusions: Real time visualization of contrast enhancement without radiation exposure is one of the main advantages of CEUS over other diagnostic modalities to differentiate ascariasis debris in the gallbladder from enhancing neoplasia. Thus, surgery and other interventions and their complications could be avoided.
\end{abstract}

Keywords: ascariasis; gallbladder; contrast enhanced ultrasound; adenoma; adenocarcinoma

\section{Introduction}

Ascariasis is one of the most common parasitic roundworm infections worldwide. Most patients with $A$. lumbricoides or $A$. suum infection are asymptomatic but need anthelminthic treatment [1]. Since worm invasion

Received 10.08.2018 Accepted 25.09.2018

Med Ultrason

2018, Vol. 20, No 4, 413-419

Corresponding author: Prof. Wen-Ping Wang

Department of Ultrasound,

Zhongshan Hospital, Fudan University

180 Fenglin Road, Shanghai, 200032, China

Phone: 86 (0)21- 64041990 - 2474

Fax: $86(0) 21$ - 64220319

E-mail: puguang61@126.com into the gallbladder (GB) is less common because of anatomical features, GB ascariasis is quite rare, constituting $2.1 \%$ of hepatobiliary ascariasis [2-7].

Conventional B-mode ultrasound (BMUS), as a noninvasive, easy and safe procedure, is currently the firstline imaging modality for the diagnosis of gallbladder disease. It has been reported to have satisfactory diagnostic accuracy in the work-up of biliary ascariasis $[8,9]$. Previously, several studies have discussed the BMUS findings of the roundworm in the gallbladder and biliary tracts $[4,8,9]$. However, limitations of BMUS arise in several differentiating types of gallbladder lesions, such as motionless stiff sludge or ascariasis debris which can generate similar BMUS images with gallbladder tumors [10]. In some complicated cases, ascariasis debris will 
give a pseudotumorous appearance [4,11], which might lead to unnecessary operations $[12,13]$. A correct characterization of GB lesions is of importance to avoid unnecessary cholecystectomies and is helpful to clinical decision-making.

In 2012, EFSUMB (European Federation of Societies for Ultrasound in Medicine and Biology) contrast enhanced ultrasound (CEUS) guidelines were introduced for the biliary system [14-16]. CEUS has been used in the current clinical guidelines and practice in the differentiation of benign or malignant GB lesions in selected cases $[14,17,18]$. CEUS of the GB can provide real-time, reproducible, multiplane imaging without compromise to patient safety or exposure to radiation $[14,19]$. However, up till now, published data about CEUS for gallbladder parasitic disorders are scarce.

The aim of our current retrospective study is to investigate the value of CEUS for differential diagnosis between ascariasis debris and adenoma/adenocarcinoma of gallbladder.

\section{Material and methods}

\section{Patients}

From July 2015 to Aug 2017, we retrospectively enrolled 35 patients with focal or solitary gallbladder lesions and underwent both BMUS and CEUS.

The inclusion criteria were as follows: 1) primary BMUS showed focal hyperechoic gallbladder lesion $(>10 \mathrm{~mm})$ not mobile in the standing position; 2$)$ referral for exclusion of malignancy by clinicians or radiologists; 3) patients who received both BMUS and CEUS examinations; and 4) all cases were confirmed by pathologic examination after surgery $(\mathrm{n}=26)$ or clinical follow-up $(\mathrm{n}=9)$.

Exclusion criteria included patient contraindications to the contrast agent, such as a history of cardiac failure, or respiratory disorders and pregnancy or lactation.

\section{D CEUS}

Conventional BMUS and CEUS examinations were performed by a single experienced radiologist (more than 10 years' experience of CEUS of the liver), who was aware of the patients' clinical history.

All ultrasound examinations were performed by an S2000 HELX OXANA unit (contrast pulse sequencing, CPS) (Siemens Healthineers, Germany), with a $3.5 \mathrm{MHz}$ $6 \mathrm{C}-1$ convex array probe and additionally with a 7.5$12 \mathrm{MHz}$ 9L4 high frequency transducer, equipped with pulse inversion imaging software in some very superficially located lesions.

All patients fasted for at least 8 hours before ultrasound examinations. Each patient underwent a complete examination of the GB and the adjacent liver under baseline BMUS before CEUS. The maximal diameter of the GB and the thickness of the GB wall were measured using BMUS. Then the BMUS features of the gallbladder lesions were assessed according to a standardized protocol (only patients with underlined criteria were included into analysis): number of lesions (solitary or multiple), size $(>10 \mathrm{~mm})$, echogenicity (hyperechoic, isoechoic, hypoechoic, mixed compared to liver tissue); location and mobility during supine and standing position (bottom, body, neck, whole GB); feature of the lesion bottom (narrow, broad), and blood flow on color flow imaging (presence or absence).

\section{Conventional CEUS procedure}

CEUS was performed on the same day after baseline BMUS. The ultrasound contrast agent used in the present study was sulfur hexafluoride (SonoVue ${ }^{\mathrm{TM}}$, Bracco, Milan, Italy), which was injected intravenously as a $2.4 \mathrm{~mL}$ bolus followed by $10 \mathrm{~mL}$ of normal sterile saline flush using a 20- Gauge peripheral intravenous cannula. A low frame rate ( $>10$ per second) and a very low mechanical index (MI), ranging from 0.05 to 0.08 , were used for real time imaging.

During CEUS procedure, the target GB lesion was placed in the center of the screen and the transducer was kept in a stable position. The target GB lesion was scanned for the following 3-5 min in order to assess its enhancement pattern from the arterial phase $(<30 \mathrm{sec})$ to the later phases $(>31 \mathrm{sec})$ [10,20-22]. The contrast enhancement of the GB lesions was evaluated including branching intramural vessels and gallbladder wall integrity. In the later phase the liver was scanned for detection of metastases.

The enhancement patterns on CEUS were classified as follows: 1) enhancement homogeneity, including homogeneous enhancement, heterogeneous enhancement or non-enhancement; 2) enhancement degree, including marked enhancement, mild enhancement or non-enhancement while comparing to that of the adjacent liver parenchyma at the time of peak intensity; 3) the intralesional enhancement during the arterial phase, including branched, linear, dotted, and none; 4) the GB wall integrity under the GB lesions (intact and destructed). Destruction of the intactness of the gallbladder wall was defined as incomplete continuity; and 5) the detection of liver metastasis during a late phase liver sweep (present or absent).

BMUS and CEUS with high frequency transducer

Afterwards, BMUS and CEUS with a high frequency transducer were performed on 9 suspicious GB lesions which were located at a very superficial location. The targeted CEUS with high frequency transducer were per- 
formed with an intravenously bolus injection of $2.4 \mathrm{ml}$ SonoVue ${ }^{\circledR}$ (Bracco, Milan, Italy) followed by $10 \mathrm{ml}$ of normal sterile saline flush. The same BMUS and CEUS imaging criteria as described for the low frequency transducer were applied. The BMUS, CDFI (color Doppler flow imaging) and CEUS imaging features were recorded and compared.

\section{Statistical analysis}

Statistical analyses were performed using SPSS15.0 for Windows (SPSS Inc., Chicago, IL). Continuous data were expressed as mean ( \pm standard deviation (Std)). The comparison between the continuous data was tested using the independent $t$ test if normal distribution was achieved; otherwise, nonparametric Mann-Whitney U test was used. McNemar's test was used to evaluate the diagnostic efficacy. $p$ values $<0.05$ were considered statistically significant.

\section{Institutional board approval}

This retrospective study was approved by the institutional review board of our institution. Informed consent was waived before CEUS examination. The procedure followed was in accordance with the Declaration of Helsinki.

\section{Results}

\section{Final diagnosis}

A total of 35 patients were finally included in this study (Table I). The final diagnoses of the 35 patients included GB adenocarcinoma $(\mathrm{n}=15)$, adenomas $(\mathrm{n}=11)$ and ascariasis debris $(\mathrm{n}=9)$.

CEUS imaging analysis were performed in all these patients. Of the 35 patients, 26 subsequently underwent surgery and the final diagnoses were proved histopathologically. For the remaining 9 patients, a final diagnosis
Table I. Baseline characteristics of patients

\begin{tabular}{ll}
\hline & $\begin{array}{l}\text { Patients } \\
(\mathbf{n = 3 5 )}\end{array}$ \\
\cline { 2 - 2 } Age (year) & \\
$\quad$ Mean \pm SD & $54 \pm 10$ \\
$\quad$ Range & $20-67$ \\
Male/female & $20 / 15$ \\
Final diagnosis established by & \\
$\quad$ surgery & 26 \\
$\quad$ follow-up & 9 \\
\hline
\end{tabular}

was confirmed by clinical infection history, laboratory data and follow-up examinations, including conventional BMUS and CDFI.

\section{BMUS}

All 35 lesions manifested as a solitary hyperechoic solid lesion in the gallbladder on conventional BMUS with no mobility in the standing position. The zigzag and multiple parallel lines echogenic were detected inside the lumen of the gallbladder in 6 patients with ascariasis.

Among the 26 neoplastic lesions, most of the benign GB adenoma lesions $(8,72.7 \%)$ were pedunculated $(\mathrm{p}=0.002)$. Blood flow on color Doppler imaging was detected in 10 neoplastic lesions (38.4\%), whereas 16 neoplastic lesions (61.6\%) and 9 non-neoplastic lesions showed no blood flow (Table II).

\section{CEUS enhancement}

After injection of $2.4 \mathrm{ml}$ ultrasound contrast agents, all solitary hyperechoic GB lesion in patients with ascariasis showed no enhancement during the whole enhancement period (fig 1, fig 2). Meanwhile, the other 26 gallbladder hyperechoic lesions showed wash-in and wash-out enhancement at certain degrees $(\mathrm{p}=0.006)$ (fig 3).

Table II. B mode ultrasound features of gallbladder lesions

\begin{tabular}{|c|c|c|c|c|}
\hline Characteristic & $\begin{array}{l}\text { Ascariasis debris } \\
(\mathrm{n}=9)\end{array}$ & $\begin{array}{l}\text { Gallbladder adenoma } \\
(\mathrm{n}=11)\end{array}$ & $\begin{array}{l}\text { Gallbladder adenocarcinoma } \\
(n=15)\end{array}$ & p value \\
\hline Diameter (mm) & $11.7 \pm 3.6$ & $12.1 \pm 3.1$ & $14.3 \pm 1.2$ & 0.41 \\
\hline $\begin{array}{l}\text { Location } \\
\text { Bottom } \\
\text { Body } \\
\text { Neck }\end{array}$ & $\begin{array}{l}1(11.1) \\
4(44.4) \\
4(44.4)\end{array}$ & $\begin{array}{l}5(45.5) \\
3(27.3) \\
3(27.3)\end{array}$ & $\begin{array}{l}3(20.0) \\
5(33.3) \\
7(46.7)\end{array}$ & $\begin{array}{l}0.53 \\
0.51 \\
0.40\end{array}$ \\
\hline $\begin{array}{l}\text { Lesion bottom } \\
\text { Narrow } \\
\text { Broad }\end{array}$ & $\begin{array}{l}0 \\
9(100)\end{array}$ & $\begin{array}{l}8(72.7) \\
3(27.3)\end{array}$ & $\begin{array}{l}0 \\
15(100)\end{array}$ & $\begin{array}{l}<0.05 \\
<0.05\end{array}$ \\
\hline $\begin{array}{l}\text { Blood flow (CFI) } \\
\text { Presence } \\
\text { Absence } \\
\text { GB wall thickness (mm) }\end{array}$ & $\begin{array}{l}0 \\
9(100) \\
2.1 \pm 0.6\end{array}$ & $\begin{array}{l}3(27.3) \\
8(72.7) \\
2.0 \pm 0.4\end{array}$ & $\begin{array}{l}7(46.7) \\
8(53.3) \\
2.4 \pm 0.3\end{array}$ & $\begin{array}{l}0.47 \\
0.81 \\
0.56\end{array}$ \\
\hline
\end{tabular}

The results are expressed as mean \pm standard deviation or number(\%); CFI - color flow imaging; GB - Gallbladder 


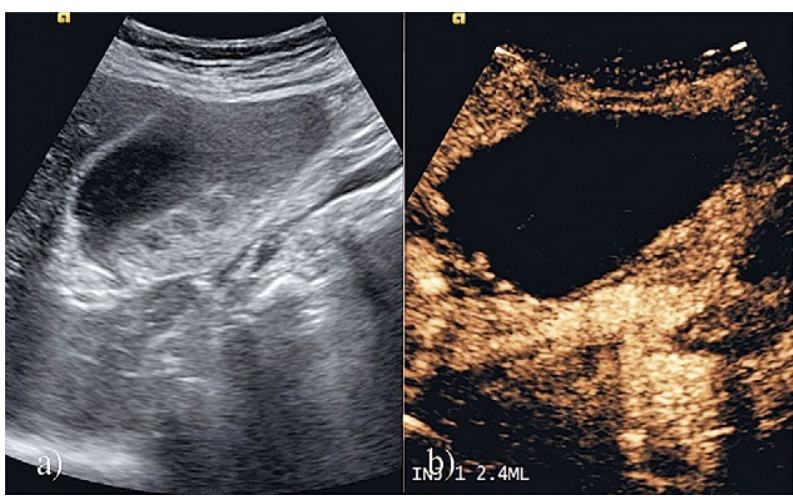

Fig 1. Gallbladder Ascariasis debris. B-mode ultrasound image evidenced a zigzag hyperechoic lesion in the gallbladder of a 57-year-old male patient (a). After injection of ultrasound contrast agents, the hyperechoic lesion showed non-enhancement during the whole arterial and late phase (b).

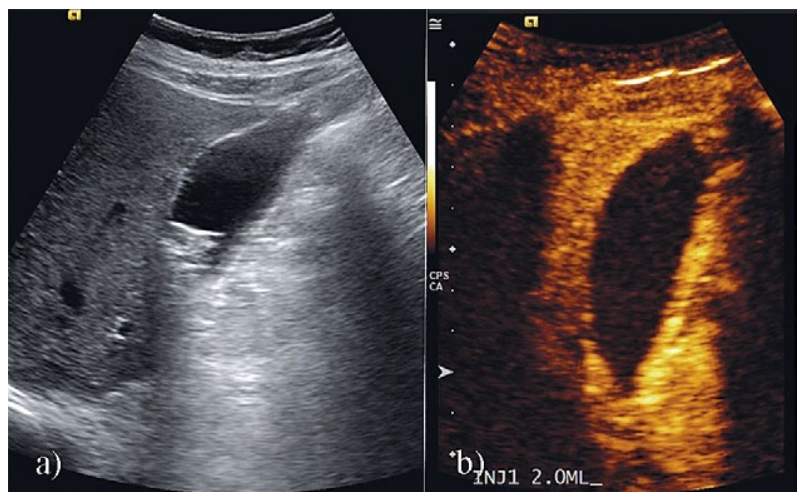

Fig 2. Gallbladder Ascariasis debris. B-mode ultrasound image evidenced a multiple parallel lines hyperechoic lesion inside the lumen of gallbladder in a 45-year-old female patient (a). After injection of ultrasound contrast agents, the hyperechoic lesion showed non-enhancement during the arterial and late phase (b).
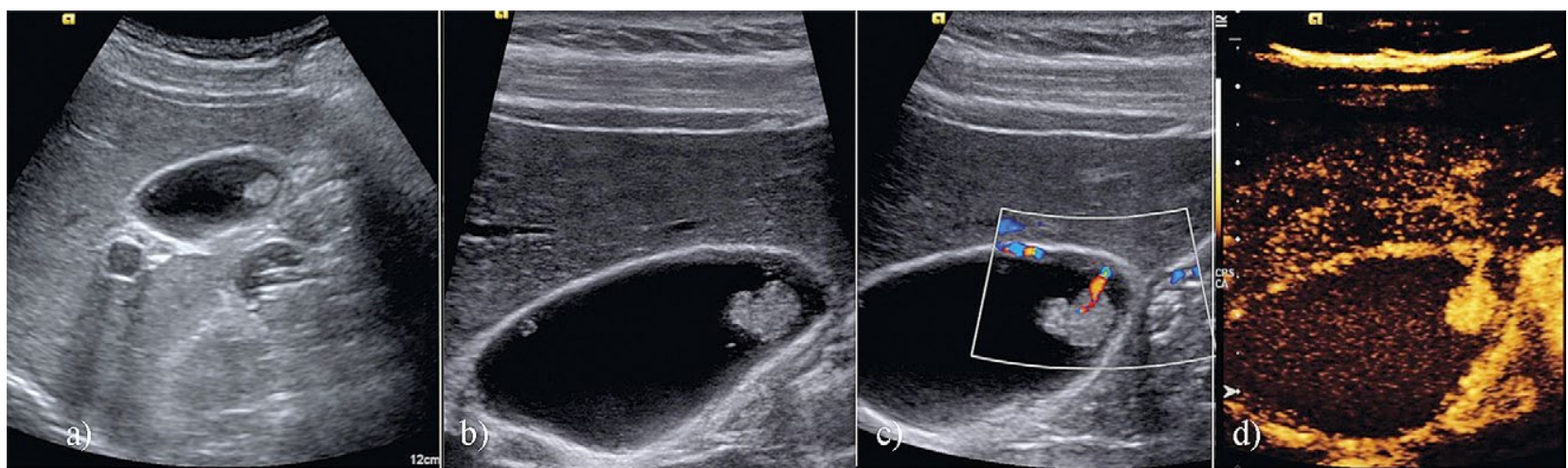

Fig 3. Gallbladder adenoma. B-mode ultrasound image detected a hyperechoic lesion inside the lumen of gallbladder in a 37-yearold male patient (a). The lesion and the gallbladder wall are clearer with a high frequency ultrasound transducer (b). Color flow imaging detected arterial flow signals inside the lesion (c). After injection of ultrasound contrast agents, the lesion showed hyperenhancement during the arterial while the continuity of the gallbladder wall was visualized (d).

Table III shows the results of CEUS enhancement images according to the gallbladder lesions' type.

\section{Special BMUS and CEUS imaging features with high frequency transducer}

While using BMUS with a high frequency transducer on 9 suspicious superficial GB lesions, zigzag and multiple parallel lines echogenic features were more obvious in $3 \mathrm{~GB}$ lesions, which were not visible by BMUS with a low frequency transducer.

On CDFI, pulsed color Doppler of arterial flow signals were detected in all 6 superficially located GB neoplastic lesions by a high frequency transducer, while only in 1 lesion by a low frequency transducer $(\mathrm{p}=0.005)$.

On CEUS destruction of the GB wall intactness was observed as an incomplete enhancement in 3 superficially located malignant GB neoplastic lesions, while the continuity of the GB wall was visualized on other 6 benign GB lesions (intact and destructed).

\section{Diagnostic efficacy of CEUS}

The overall sensitivity, specificity and diagnostic accuracy for the correct characterization of ascariasis debris of gallbladder were significantly improved by CEUS compared to BMUS (sensitivity: 98.8 vs $32.9 \%$, $\mathrm{p}<0.05$; specificity: 96.5 vs $17.4 \%, \mathrm{p}<0.05$; accuracy: $100 \%$ vs $66.6 \%, \mathrm{p}<0.01$, McNemar's test).

\section{Discussions}

Ascariasis is caused by Ascaris lumbricoides roundworms and is one of the most common helminthic infections in human beings. The adult worm is usually seen in the small intestine, mainly in the jejunum. GB ascariasis is a very rare presentation. Sometimes the worm enters 
Med Ultrason 2018; 20(4): 413-419

Table III. Contrast enhancement features of 35 gallbladder lesions

\begin{tabular}{llll}
\hline Characteristic & $\begin{array}{l}\text { Ascariasis debris } \\
(\mathbf{n = 9 )}\end{array}$ & $\begin{array}{l}\text { Gallbladder adenoma } \\
(\mathbf{n = 1 1})\end{array}$ & $\begin{array}{l}\text { Gallbladder adenocarcinoma } \\
(\mathbf{n}=\mathbf{1 5})\end{array}$ \\
\hline Arterial phase enhancement & & $3(27.3)$ & $12(80)$ \\
$\quad$ Hyperenhanced & 0 & $8(72.7)$ & $3(20)$ \\
$\quad$ Isoenhanced & 0 & 0 & 0 \\
$\quad$ Non-enhanced & $9(100)$ & & $2(13.3)$ \\
Enhancement patterns in arterial phase & & $10(90.9)$ & $13(86.7)$ \\
$\quad$ Homogeneous enhancement & 0 & $1(9.1)$ & 0 \\
$\quad$ Inhomogeneous enhancement & 0 & 0 & \\
$\quad$ Non-enhancement & $9(100)$ & & $5(33.3)$ \\
Interruption of gallbladder wall & & $11(100)$ & $10(66.7)$ \\
$\quad$ Continuous & $9(100)$ & 0 & \\
$\quad$ Interrupted & 0 & & \\
\hline
\end{tabular}

The results are expressed as a number (\%)

into the common bile duct, passing through the papilla of Vater present in the second part of duodenum and then into the gallbladder lumen through the cystic duct [5].

Preoperative radiologic diagnosis is very important, as the management of neoplastic lesions (surgery) and non-neoplastic ascariasis debris of the gallbladder (medical treatment) is completely different. Large tumor-like ascariasis debris lesions should be differentiated from a GB adenoma or carcinoma before operation.

BMUS is the initial modality of choice for the diagnosis of gallbladder ascariasis [23]. The live worm might present as tubular, linear, parallel, non-shadowing echogenic lines inside the gallbladder lumen. On BMUS, coiling, zigzag and non-directional movements features could be visualized [5]. Common typical ultrasound features of ascariasis have been described as linear or curvilinear thick, echogenic, non-shadowing structures with central anechoic longitudinal tubes $[1,4,9]$. However, the application of BMUS in the GB is limited to differentiating tumor and pseudotumorous appearance in gallbladder lesions, such as motionless stiff sludge or ascariasis debris [10,24].

Previously there were several clinical studies on the application of CEUS in differentiating between benign and malignant gallbladder lesions. CEUS has been proved to be a sensitive and accurate diagnostic technique in clinical settings [10,12-14,20,22,25-27]. Compared with the liver, CEUS of the gallbladder shows a different hemodynamic behavior. The gallbladder is perfused only by the cystic artery without portal vein branches [17,18,25,26]. All gallbladder tumors, benign or malignant, are supplied by arterial vessels from branches of the cystic artery [14$16,28,29]$. It has been reported that hyperenhancement in the arterial phase was present in $84.8 \%$ of carcinomas and in $70.2 \%$ of benign lesions $(92.2 \%$ cholesterol polyps, $77.8 \%$ adenomas and $85.5 \%$ chronic cholecystitis)
$[10,28,30]$. According to the most recent published EFSUMB guideline and recommendations for the clinical practice of CEUS, differentiation between benign and malignant gallbladder tumors cannot be made by hyperenhancement during the arterial phase as gallbladder cancers $(85 \%)$ and benign gallbladder diseases $(70 \%)$, both showing hyper-enhancement [17,18,31,32].

On CEUS, microbubble contrast agents remain entirely intravascular. In the differentiation between GB cancer and motionless biliary sludge CEUS has a sensitivity of $100 \%[10,19,30,33)]$. Since irregular intraluminal GB ascariasis debris is avascular, therefore, in our series, no enhancement is observed at any time during CEUS. CEUS provides an advantage over conventional BMUS by utilizing the presence or absence of vascularity.

Accurate description of the GB wall involvement pattern can modify patient clinical management. The destruction of the gallbladder wall integrity on CEUS is the CEUS feature highly suggestive of malignancy and highly suggestive of gallbladder cancer $[10,13,17,18]$. Anyway, in GB ascariasis debris, the outer gallbladder layer must appear sharp and a clear cleavage plane with the liver must always be present [34]. In our study, the use of a high frequency transducer enables more clear identification and characterization of focal or diffuse GB wall thickening in most cases. While using a high frequency transducer for CEUS, disruption of the gallbladder wall integrity appears in 3 of GB carcinomas and in none of the benign lesions (specificity 100\%).

In clinical practice, GB adenoma may involve the gallbladder fundus, which is usually unsatisfactorily evaluated by means of the classical trans-abdominal convex transducers [34-36]. Meanwhile, in our study CEUS with a high frequency transducer improves the visualization of the vascular fundus of GB adenomas. We suggested that every suspicious lesion in the superficial area of 
the GB should be further investigated by means of higher frequency linear probes for better characterization.

Our retrospective study has some limitations, such as the limited number of GB lesions included. Also, most of GB ascariasis debris cases were confirmed by signs of clinical infection history, treatment and follow-up examination.

\section{Conclusion}

CEUS can be used to differentiate between GB tumors and GB ascariasis debris with a higher confidence compared with BMUS. With its superior contrast resolution, CEUS performed with a high frequency transducer is helpful to detect GB wall destruction beneath a solid lesion and better visualization of the vascular fundus of GB adenomas, which might also improve diagnostic confidence.

Acknowledgment: Supported by the National Natural Science Foundation of China (No. 81571676, No.81371577, No.81501471)

\section{Conflict of interest: none}

\section{References}

1. Al Absi M, Qais AM, Al Katta M, Gafour M, Al-Wadan AH. Biliary ascariasis: the value of ultrasound in the diagnosis and management. Ann Saudi Med 2007;27:161-165.

2. Dietrich CF, Sharma M, Chaubal N, et al. Ascariasis imaging: pictorial essay. Z Gastroenterol 2017;55:479-489.

3. Filice C, Marchi L, Meloni C, Patruno SF, Capellini R, Bruno R. Ultrasound in the diagnosis of gallbladder ascariasis. Abdom Imaging 1995;20:320-322.

4. Lynser D, Marbaniang E. Hepatobiliary and pancreatic ascariasis-sonographic pictorial essay on four pediatric cases with acute biliary colic. Emerg Radiol 2016;23:197-200.

5. Khanduri S, Parashari UC, Agrawal D, Bhadury S. Ascariasis of gallbladder: a rare case report and a review of the literature. Trop Doct 2014;44:50-52.

6. Dietrich CF, Chichakli M, Hirche TO, et al. Sonographic findings of the hepatobiliary-pancreatic system in adult patients with cystic fibrosis. J Ultrasound Med 2002;21:409416.

7. Danaci M, Belet U, Selcuk MB, Akan H, Bastemir M. Ascariasis of the gallbladder: radiological evaluation and follow-up. Pediatr Radiol 1999;29:80.

8. Das AK. Hepatic and biliary ascariasis. J Glob Infect Dis 2014;6:65-72.

9. Lynser D, Handique A, Daniala C, Phukan P, Marbaniang E. Sonographic images of hepato-pancreatico-biliary and intestinal ascariasis: A pictorial review. Insights Imaging 2015;6:641-646.
10. Xie XH, Xu HX, Xie XY, et al. Differential diagnosis between benign and malignant gallbladder diseases with real-time contrast-enhanced ultrasound. Eur Radiol 2010;20:239-248.

11. Foster T, Hamper U. Biliary Ascariasis: A Case Report. U1trasound Q 2015;31:232-233.

12. Gerstenmaier JF, Hoang KN, Gibson RN. Contrast-enhanced ultrasound in gallbladder disease: a pictorial review. Abdom Radiol (NY) 2016;41:1640-1652.

13. Liu LN, Xu HX, Lu MD, et al. Contrast-enhanced ultrasound in the diagnosis of gallbladder diseases: a multi-center experience. PLoS One 2012;7:e48371.

14. Piscaglia F, Nolsoe C, Dietrich CF, et al. The EFSUMB Guidelines and Recommendations on the Clinical Practice of Contrast Enhanced Ultrasound (CEUS): update 2011 on non-hepatic applications. Ultraschall Med 2012;33:33-59.

15. Claudon M, Dietrich CF, Choi BI, et al. Guidelines and good clinical practice recommendations for Contrast Enhanced Ultrasound (CEUS) in the liver - update 2012: A WFUMB-EFSUMB initiative in cooperation with representatives of AFSUMB, AIUM, ASUM, FLAUS and ICUS. Ultrasound Med Biol 2013;39:187-210.

16. Claudon M, Dietrich CF, Choi BI, et al. Guidelines and good clinical practice recommendations for contrast enhanced ultrasound (CEUS) in the liver--update 2012: a WFUMBEFSUMB initiative in cooperation with representatives of AFSUMB, AIUM, ASUM, FLAUS and ICUS. Ultraschall Med 2013;34:11-29.

17. Sidhu PS, Cantisani V, Dietrich CF, et al. The EFSUMB Guidelines and Recommendations for the Clinical Practice of Contrast-Enhanced Ultrasound (CEUS) in Non-Hepatic Applications: Update 2017 (Short Version). Ultraschall Med 2018;39:154-180.

18. Sidhu PS, Cantisani V, Dietrich CF, et al. The EFSUMB Guidelines and Recommendations for the Clinical Practice of Contrast-Enhanced Ultrasound (CEUS) in Non-Hepatic Applications: Update 2017 (Long Version). Ultraschall Med 2018;39:e2-e44.

19. Meacock LM, Sellars ME, Sidhu PS. Evaluation of gallbladder and biliary duct disease using microbubble contrast-enhanced ultrasound. Br J Radiol 2010;83:615-627.

20. Tang S, Wang Y, Wang Y. Contrast-enhanced ultrasonography to diagnose gallbladder perforation. Am J Emerg Med 2013;31:1240-1243.

21. Fei X, Lu WP, Luo YK, et al. Contrast-enhanced ultrasound may distinguish gallbladder adenoma from cholesterol polyps: a prospective case-control study. Abdom Imaging 2015;40:2355-2363.

22. Sun LP, Guo LH, Xu HX, et al. Value of contrast-enhanced ultrasound in the differential diagnosis between gallbladder adenoma and gallbladder adenoma canceration. Int J Clin Exp Med 2015;8:1115-1121.

23. Chakrabarti I, Giri A, De A, Roy AC. Radio-pathological diagnosis of hepatobiliary ascariasis: A rare entity. J Cytol 2011;28:114-116.

24. Joo I, Lee JY, Kim JH, et al. Differentiation of adenomyomatosis of the gallbladder from early-stage, wall-thick- 
ening-type gallbladder cancer using high-resolution ultrasound. Eur Radiol 2013;23:730-738.

25. Zhuang B, Li W, Wang W, et al. Contrast-enhanced ultrasonography improves the diagnostic specificity for gallbladder-confined focal tumors. Abdom Radiol (NY) 2018;43:1134-1142.

26. Badea R, Zaro R, Opincariu I, Chiorean L. Ultrasound in the examination of the gallbladder - a holistic approach: grey scale, Doppler, CEUS, elastography, and 3D. Med U1trason 2014;16:345-355.

27. Zheng SG, Xu HX, Liu LN, et al. Contrast-enhanced ultrasound versus conventional ultrasound in the diagnosis of polypoid lesion of gallbladder: a multi-center study of dynamic microvascularization. Clin Hemorheol Microcirc 2013;55:359-374.

28. Numata K, Oka H, Morimoto M, et al. Differential diagnosis of gallbladder diseases with contrast-enhanced harmonic gray scale ultrasonography. J Ultrasound Med 2007;26:763-774.

29. Chen LD, Huang Y, Xie XH, et al. Diagnostic nomogram for gallbladder wall thickening mimicking malignancy: using contrast-enhanced ultrasonography or multi-detector com- puted tomography? Abdom Radiol (NY) 2017;42:24362446.

30. Sparchez Z, Radu P. Role of CEUS in the diagnosis of gallbladder disease. Med Ultrason 2012;14:326-330.

31. Shapira-Rootman M, Mahamid A, Reindorp N, Nachtigal A, Zeina AR. Diagnosis of gallbladder perforation by ultrasound. Clin Imaging 2015;39:827-829.

32. Xu JM, Guo LH, Xu HX, et al. Differential diagnosis of gallbladder wall thickening: the usefulness of contrastenhanced ultrasound. Ultrasound Med Biol 2014;40:27942804.

33. Inoue T, Kitano M, Kudo M, et al. Diagnosis of gallbladder diseases by contrast-enhanced phase-inversion harmonic ultrasonography. Ultrasound Med Biol 2007;33:353-361.

34. Bonatti M, Vezzali N, Lombardo F, et al. Gallbladder adenomyomatosis: imaging findings, tricks and pitfalls. Insights Imaging 2017;8:243-253.

35. Mellnick VM, Menias CO, Sandrasegaran K, et al. Polypoid Lesions of the Gallbladder: Disease Spectrum with Pathologic Correlation-Erratum. Radiographics 2015;35:1316.

36. Aloia TA, Jarufe N, Javle M, et al. Gallbladder cancer: expert consensus statement. HPB (Oxford) 2015;17:681-690. 